Full length article

\title{
Facile preparation of mussel-inspired antibiotic-decorated titanium surfaces with enhanced antibacterial activity for implant applications
}

\author{
Jae Seo Lee ${ }^{\mathrm{a}, 1}$, Sang Jin Lee ${ }^{\mathrm{b}, 1}$, Seok Bin Yang ${ }^{\mathrm{c}}$, Donghyun Lee ${ }^{\mathrm{b}, \mathrm{d}}$, Haram Nah ${ }^{\mathrm{a}}$, \\ Dong Nyoung Heo ${ }^{\mathrm{b}}$, Ho-Jin Moon ${ }^{\mathrm{b}}$, Yu-Shik Hwang ${ }^{\mathrm{c}}$, Rui L. Reis ${ }^{\mathrm{b}, \mathrm{e}}$, Ji-Hoi Moon ${ }^{\mathrm{c}, *, 2}$, \\ Il Keun Kwon ${ }^{\mathrm{b}, *, 2}$ \\ ${ }^{a}$ Department of Dentistry, Graduate School, Kyung Hee University, 26 Kyungheedae-ro, Dongdaemun-gu, Seoul 02447, Republic of Korea \\ ${ }^{\mathrm{b}}$ Department of Dental Materials, School of Dentistry, Kyung Hee University, 26 Kyungheedae-ro, Dongdaemun-gu, Seoul 02447, Republic of Korea \\ ${ }^{\mathrm{c}}$ Department of Maxillofacial Biomedical Engineering and Institute of Oral Biology, School of Dentistry, Kyung Hee University, 26 Kyungheedae-ro, Dongdaemun-gu, Seoul \\ 02447, Republic of Korea \\ d Laboratory Animal Center, Daegu-Gyeongbuk Medical Innovation Foundation, 80 Cheombok-ro, Dong-gu, Daegu 41061, Republic of Korea \\ e $3 B$ 's Research Group, I3Bs - Research Institute on Biomaterials, Biodegradables and Biomimetics of University of Minho, Headquarters of the European Institute of \\ Excellence on Tissue Engineering and Regenerative Medicine, AvePark - Parque de Ciência e Tecnologia, Zona Industrial da Gandra, 4805-017 Barco/Guimarães, Portugal.
}

\section{A R T I C L E I N F O}

\section{Keywords:}

Titanium implant

Polydopamine

Ceftazidime

Biocompatibility

Antibacterial property

\begin{abstract}
A B S T R A C T
Titanium implants (Ti) have been widely used in several medical fields. In clinical practice, Ti can become contaminated with bacteria through a variety of mechanisms. This contamination can lead to implant failure and serious infections. In this study, we aimed to develop a new, hybrid Ti with good biocompatibility and antibacterial properties by immobilizing ceftazidime (CFT) onto the Ti surface through polydopamine (PDA) and polyethyleneimine (PEI) chemistry. Hybrid Ti was confirmed by assessing the cell proliferation of human adipose-derived stem cells using a cell counting. The biofilm formation across the Ti surface of two bacterial strains associated with nosocomial infections, Pseudomonas aeruginosa and methicillin-resistant Staphylococcus aureus, was evaluated by scanning electron microscopy. The viability of the bacteria exposed to Ti surface was evaluated by cell counting. Our results clearly demonstrate that the bacterial biofilm formation as well as bacterial viability was significantly reduced on the hybrid $\mathrm{Ti}$ as compared to the control, Ti alone. Collectively, the Ti surface was successfully modified to form the hybrid Ti exhibiting good biocompatibility and antibacterial properties through PDA, PEI, and CFT grafting. Within the limitations of this in vitro study, we conclude that the hybrid Ti may be useful for successful implant treatment.
\end{abstract}

\section{Introduction}

Titanium implants (Ti) are commonly used for orthopedic or dental surgeries. This substrate exhibits relatively good corrosion resistance, excellent fatigue strength, and biocompatibility [1]. Due to these advantages, Ti has been widely applied for use as an implant material. For these reasons, $\mathrm{Ti}$ is an attractive metal substrate for biomedical applications [2]. In the clinical field, Ti failure sometimes occurs due to contamination by contact with surgical instruments, gloves, and air in the operating room which subsequently leads to infection [3]. The number of these infections is relatively high due to the high number of $\mathrm{Ti}$ implantations and presents a significant challenge in healthcare.
Several microorganisms are known to be involved in osteitis, periodontitis, osteomyelitis, and peri-implantitis, which can cause complications after implantation [4]. The resulting infections can lead to tissue destruction including bleeding and suppuration, which can result in loss of the supporting bone [5]. These infections can even be fatal [6].

To overcome these problems, many researchers have applied antibiotic agents using surface treatment strategies [3]. These formal methods prevent severe bacterial contamination with immediate response [7]. One of the ways for anchoring antibiotics on bare Ti is high temperature polymerization. However, it is difficult for most antibiotic drugs to survive this process and remain active for treatment [8]. Furthermore, many surface modifications use organic solvents which

\footnotetext{
* Corresponding authors.

E-mail addresses: prudence75@khu.ac.kr (J.-H. Moon), kwoni@khu.ac.kr (I.K. Kwon).

${ }^{1}$ These authors equally contributed to this work.

${ }^{2}$ These corresponding authors have made equal contributions to this work.
} 
still remain as residues on the surface and these residues have toxic effects towards cells $[8,9]$. Therefore, there is a need in biomedical engineering to develop a more facile approach which does not use high temperatures or organic solvents.

One facile surface treatment tool is the use of polydopamine (PDA), a natural material derived from mussel which has been widely investigated as a bioinspired surface modification material in biomedical engineering [10]. PDA has been used to perform surface treatment of various substrates as it has high binding strength under basic aqueous conditions [11]. In a previous report, Yuan et al. [12] demonstrated the development of stainless-steel hybrids using catechol groups of coated substrate. These hybrids were used to anchor functional biomolecules bearing carboxylic acid moieties, including antibacterial and antifouling agents. As is well known, the PDA layer can bind a biomolecule having carboxyl groups [13,14]. However, although PDA has some amine groups in its chemical structure, it possesses a limited grafting capacity for antibiotics [15]. In order to obtain amine-rich Ti surface, we employed polyethyleneimine (PEI) chemistry. PEI is a group of amine-rich cationic polymers which has been widely used as a modification tool in biomedical engineering [16]. Thus, we assumed that the introduction of PEI onto PDA modified Ti substrates allows for an amine-rich surface [17].

In this study, we speculated that amine-rich $\mathrm{Ti}$ can facilitate the grafting of large amounts of antibiotics containing carboxyl groups. We designed both biocompatible and antibacterial Ti hybrids through immobilization of ceftazidime (CFT) on PDA/PEI coated Ti substrates (Fig. 1). CFT is a third-generation cephalosporin antibiotic that has a broad spectrum of activity. It has good clinical activity against gramnegative organisms, especially Pseudomonas aeruginosa. Additionally,
CFT presents low toxicity towards human cells while effectively preventing the growth of bacteria [18].

The major aim of this study was to prepare Ti substrates having potent antimicrobial activity via facile methods and evaluate these for prevention of the initial period of infection and early implant failures. The resultant products were analyzed by ultraviolet-visible absorption spectra (UV), transmission electron microscopy (TEM), scanning electron microscopy (SEM), X-ray photoelectron spectroscopy (XPS), and atomic force microscopy (AFM). Additionally, in vitro cell proliferation was investigated. Cell morphology was confirmed by SEM, live/dead assays, and confocal laser scanning microscopy (CLSM). Finally, in vitro antibacterial tests were performed against pathogenic bacteria including Pseudomonas aeruginosa (P. aeruginosa) and Staphylococcus aureus ( $S$. aureus). Thus, bacterial eradication by the Ti surface modification was effectively confirmed by two different methods.

\section{Materials and methods}

\subsection{Materials}

Titanium discs meeting ASTM F67 grade 4 were obtained from Biotem (Sungnam, Republic of Korea). 1-Ethyl-3-(3-dimethyl aminopropyl) carbodiimide hydrochloride (EDC) and N-hydroxysuccinimide (NHS) were purchased from TCI (Tokyo Kasie Kogyo Co. Ltd., Japan). Deionized distilled water (DW) was produced by an ultra-pure water system (Puris-Ro800, Bio Lab Tech., Korea). Human Adipose Tissuederived MSC, CEFOgroTM ADMSC Human Adipose tissue-derived MSC Growth Medium, and CEFOgroTM ADMSC Supplement (10\% FBS, $0.02 \%$ Penicillin \& Streptomycin) were purchased from CEFO CO.

\section{Step 1 : Modification of titanium surface}

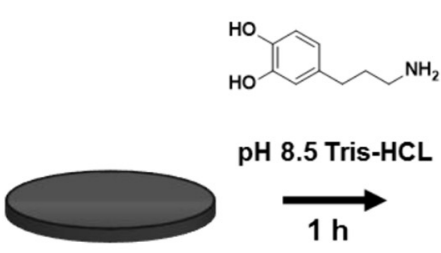

Ti

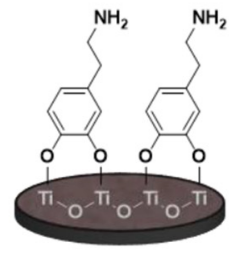

Ti-PDA

(Ti-D)
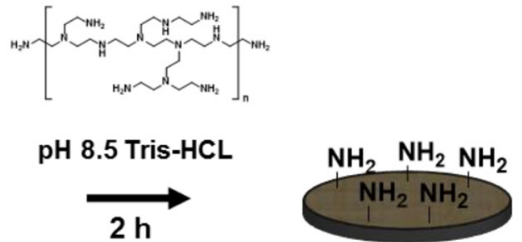

Ti-PDA-PEI

(Ti-DP)
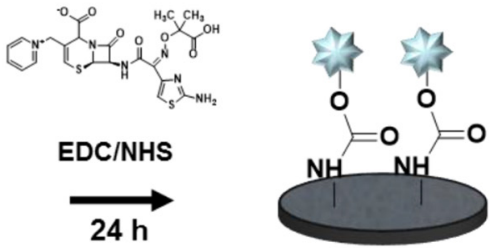

Ti-PDA-PEI-CFT (Ti-DPC)

\section{Step 2 : In vitro evaluation}
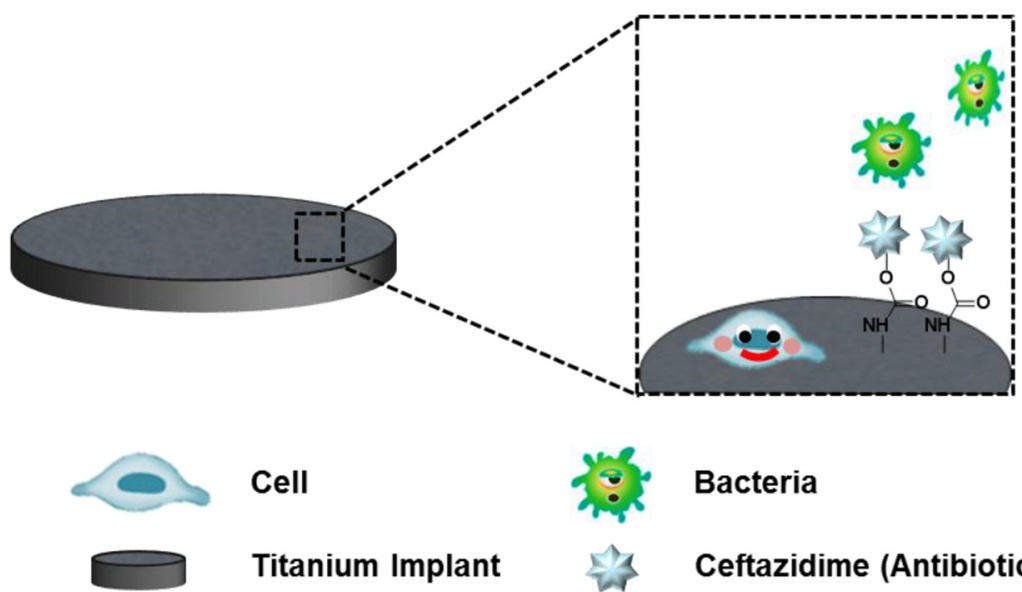

Cell

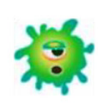

Bacteria

Titanium Implant

Ceftazidime (Antibiotic)

Fig. 1. Schematic illustration of antibacterial bio-functionalization of titanium implant. 
Dopamine hydrochloride, polyethyleneimine solution (PEI) and ceftazidime hydrate (CFT) were purchased from Sigma-Aldrich (St Louis, MO, USA). All reagents and solvents were used as received without further purification.

\subsection{Preparation of PDA and PEI coated Ti substrate}

Specific surface treatment procedure was performed following an our recent previously reported with some modifications [19]. Briefly, Ti discs were washed 3 times and cleaned in an ultra-sonicator for $10 \mathrm{~min}$ using hexane, acetone, ethanol and distilled water, sequentially, prior to use [20]. And then, Ti was immersed in a dopamine hydrochloride solution $(2 \mathrm{mg} / \mathrm{ml}$ in $10 \mathrm{mM}$ of Tris buffer, $\mathrm{pH} 8.5)$ at room temperature. After $3 \mathrm{~h}$, the PDA-coated Ti was washed with deionized water. The cleaned products were then vortexed in Tris-HCL and this was stirred (130 RPM) overnight. After that, PDA-coated Ti was incubated in $2 \mathrm{mg} / \mathrm{ml}$ of PEI solution (Sigma-Aldrich 482595, average Mw 1300, $50 \mathrm{wt} \%$ in $\mathrm{H}_{2} \mathrm{O}$ in Tris-HCL ( $\left.40 \mathrm{ml}, 10 \mathrm{mM}, \mathrm{pH} 8.5\right)$ ) at room temperature for $2 \mathrm{~h}$. The Ti substrates were then dried at room temperature.

\subsection{Immobilization of CFT decorated Ti substrate}

The amine-modified Ti substrates were decorated with CFT using EDC/NHS carbodiimide chemistry. Briefly, $1 \mathrm{mg} / \mathrm{ml} \mathrm{CFT} \mathrm{dissolved} \mathrm{in}$ $50 \mathrm{mM}$ EDC $(191.7 \mathrm{mg})$ and $25 \mathrm{mM}$ NHS $(57.545 \mathrm{mg})$ was prepared in $20 \mathrm{ml}$ of PBS. After $30 \mathrm{~min}$, the coated Ti discs were lowered into this solution and the reaction was allowed to proceed at RT for 3 days. After 3 days, the developed Ti discs were thoroughly washed with DW and dried at RT.

\subsection{Surface charge measurement of developed Ti substrate}

The surface charge potential of the developed Ti was further characterized by zeta potential. Each sample was soaked in $1 \mathrm{ml}$ of DW overnight at $37^{\circ} \mathrm{C}$ and the sample was injected into a flow cell. The surface charge was measured with a Malvern Instruments Zetasizer Nano S 90 (ZEN1690, UK). All values were measured in triplicates at a room temperature.

\subsection{Quantification of amine and carboxyl groups on Ti surfaces using colorimetric assays}

The density of the amine groups of each sample was measured using an Acid Orange II assay. All samples were immersed in $1.5 \mathrm{ml}$ of dye solution $(14 \mathrm{mg} / \mathrm{ml})$ in an acidic solution $(\mathrm{pH} 3$ with $1 \mathrm{M} \mathrm{HCl}$ ) for $30 \mathrm{~min}$ at $40^{\circ} \mathrm{C}$. The samples were then washed three times using the acidic solution $(\mathrm{pH} 3)$ to remove unbound dye. Once air-dried, the samples were immersed in $1 \mathrm{ml}$ of alkaline solution (pH 12 with a $1 \mathrm{M}$ $\mathrm{NaOH}$ solution). In order to release the adsorbed Acid Orange II, the $\mathrm{pH}$ was adjusted to 3 by adding $1 \% \mathrm{v} / \mathrm{v}$ of $12.3 \mathrm{M} \mathrm{HCl}$. The absorbance of the solution was then measured at $484 \mathrm{~nm}$. After CFT grafting, the number of carboxylic groups grafted on Ti-CFT was determined using the toluidine blue (TBO) assay. Each sample was incubated in $1 \mathrm{ml}$ of TBO solution ( $\mathrm{pH} \mathrm{10)}$ and then shaken for $15 \mathrm{~min}$ at $40^{\circ} \mathrm{C}$. Afterwards, the supernatant was removed, and the stained samples were washed under shaking conditions with $\mathrm{NaOH}$ solution ( $\mathrm{pH} 10$ ). Subsequently, $50 \%$ acetic acid was added to remove the TBO dye from the carboxyl groups from each sample for $30 \mathrm{~min}$. The TBO absorption was measured at $625 \mathrm{~nm}$.

\subsection{Release profile of CFT}

To study CFT release, the samples were immersed in vials containing $2 \mathrm{ml}$ of PBS solution at $\mathrm{pH} 5.5, \mathrm{pH} 6.5$ and $\mathrm{pH} 7.4$ in an incubator at $37^{\circ} \mathrm{C}$ under constant shaking at $100 \mathrm{rpm}$. At each time point, $1 \mathrm{ml}$ was extracted and refilled with fresh PBS $(1 \mathrm{ml})$. The concentration of CFT was determined by measuring the absorbance at $254 \mathrm{~nm}$ using a UV spectrophotometer (UV 1650-PC; SHIMADZU, Japan). The CFT concentration was determined based on a calibration curve for CFT in the range of $0.001,0.005,0.01,0.05,0.5,1 \mathrm{mg}$ in PBS (R2 = 0.99). All release studies were carried out in triplicate. The cumulative drug concentration was calculated and plotted against time to determine drug release profiles.

\subsection{In vitro ADSCs adhesion and proliferation tests}

To evaluate the cytotoxicity of modified Ti, ADSCs at a density of $2 \times 10^{4}(100 \mu \mathrm{l})$ were cultured on each scaffold sample in a 48-well tissue culture plate in Human Adipose tissue-derived MSC growth medium. After 1,3 and 7 days of culture at $37 \mathrm{C}$ in a $5 \% \mathrm{CO}_{2}$ atmosphere, $100 \mu$ of CCK (Dojindo Laboratories Kumamoto, Japan) solution was added into each well and the plate was incubated for $2 \mathrm{~h}$. The absorbance was measured at $450 \mathrm{~nm}$ using a Benchmark Plus microplate spectrophotometer (Bio-Rad, BR170-6930). For the confirmation of cell proliferation, the cells were visualized using a live/dead assay utilizing Calcein-AM (Thermo Scientific, Waltham, MA) to stain the cytoplasm of live cells (green) and ethidium homodimer-2 (Thermo Scientific, Waltham, MA) for staining the nuclei acid of dead cells (red). Stained cells were then observed by confocal laser scanning microscopy (CLSM, Eclipse E600W, Nikon, Tokyo, Japan) and the images were assayed using Nikon EZ-C1 software. For F-actin staining, the proliferated cells were fixed with $4 \%$ formaldehyde solution at room temperature for $20 \mathrm{~min}$. They were then treated with $1 \mathrm{ml}$ of rhodamine phalloidin (1:200) and 4',6-Diamidino-2-phenylindole (DAPI) (1:1000) solution at $37^{\circ} \mathrm{C}$ for $30 \mathrm{~min}$. The wells were washed with PBS to remove the free remnants. After the removal of supernatant, the images were also obtained by confocal laser scanning microscope (CLSM, Eclipse E600W, Nikon, Tokyo, Japan).

\subsection{Bacterial biofilm formation}

All bacterial strains used in this study were purchased from the American Type Cultures Collection (Manassas, VA). Pseudomonas aeruginosa ATCC 27853 and methicillin-resistant Staphylococcus aureus ATCC 700787 were grown in Mueller-Hinton broth at $37^{\circ} \mathrm{C}$ aerobically. Each bacterial strain grown to exponential phase was adjusted to an optical density at $600 \mathrm{~nm}\left(\mathrm{OD}_{600}\right)$ of 0.1 with new broth. Each Ti specimen was placed in each well of 24-well polystyrene plate containing the adjusted bacterial suspension, then the plate was incubated at $37^{\circ} \mathrm{C}$ aerobically, as described previously [21,22]. After $24 \mathrm{~h}$, the specimens were removed and the bacterial biofilm formed on Ti surface was washed with physiological saline, then fixed with $2.5 \%$ glutaraldehyde in $0.1 \mathrm{M}$ phosphate buffer for $1 \mathrm{~h}$ at room temperature and rinsed three times for $10 \mathrm{~min}$ each with distilled water. The biofilms were subsequently fixed with $1 \% \mathrm{OsO}_{4}$ in $0.1 \mathrm{M}$ phosphate buffer for $1 \mathrm{~h}$, followed by rinsing with distilled water. The fixed biofilms were dehydrated in successive ethanol-water mixtures with increasing ethanol concentrations of $25 \%, 50 \%$, and $75 \%(\mathrm{v} / \mathrm{v} \%)$ for $10 \mathrm{~min}$ each, then twice in pure ethanol for $10 \mathrm{~min}$ each. The samples were dried by critical-point drying and coated with gold using a sputter-coater (IB-3; Eiko, Tokyo, Japan). The biofilms were observed using a scanning electron microscope (SEM, Hitachi S-4700, Japan) at an acceleration voltage of $15 \mathrm{kV}$.

\subsection{Bacterial viability}

Each bacterial strain grown to exponential phase was adjusted to the final concentrations of $10^{6}$ colony forming units per milliliter $(\mathrm{CFU} / \mathrm{ml})$ using sterile phosphate buffered saline (PBS, pH 7.4). Then, $100 \mu \mathrm{l}$ of the diluted bacterial suspension was dropped onto the surface of each specimen. At appropriate time intervals, $100 \mu \mathrm{l}$ of sterile PBS was added onto the bacterial inoculation on the disc and mixed by pipetting. Three $10 \mathrm{ul}$ aliquots were removed from the mixed suspension, spot 
inoculated (i.e., $10 \mathrm{ul}$ in 3 drops) on Mueller-Hinton agar, then incubated at $37^{\circ} \mathrm{C}$ for $24 \mathrm{~h}$. These were subsequently imaged.

\subsection{Analysis equipments}

Developed Ti discs were observed using a scanning electron microscope (SEM, Hitachi S-4700, Japan) at an acceleration voltage of $15 \mathrm{kV}$. All samples were dried at room temperature, and then sputtercoated with gold using an IB-3 coater (Eiko, Japan) for $10 \mathrm{~min}$. The images were evaluated using image analysis software (EyeviewAnalyzer Digiplus, Korea). Atomic force microscope (AFM) images and roughness were obtained by with a PUCOStation STD (NANOS ${ }^{\circledR}$ AFM system, NanoInk, Inc., USA) operated in dynamic mode with a scanner (NANOS $^{\circledR} 100$ ) at room temperature. To evaluate the hydrophilic properties of the Ti surface, water contact angles were measured using the sessile drop method and a video contact angle instrument (Phoenix 150; SEO, Seoul, Korea). For this process, an 8- $\mu 1$ distilled water droplet was applied at room temperature. The contact angle was calculated as the angle between the Ti surface and the tangent of the liquid on the $\mathrm{Ti}$ surface using the AutoFAST algorithm and image analysis software. These experiments were repeated in triplicate. The functionalized chemistry compositions of each Ti surface were investigated by X-ray photoelectron spectroscopy (XPS, Thermo Electron Manufacturing Ltd., UK). These experiments were performed with a K-Alpha instrument (Thermo Electron, UK) to evaluate the surface chemistry of the treated Ti. The survey scans were measured from $0 \mathrm{eV}$ to $1300 \mathrm{eV}$.

\subsection{Statistical analysis}

At least three samples for each experimental condition were used. Statistical comparisons were carried out using t-test, one-way ANOVA, and two-way ANOVA tests. Statistically significant values are defined as ${ }^{*} \mathrm{p}<0.05,{ }^{* *} \mathrm{p}<0.01,{ }^{* * *} \mathrm{p}<0.001,{ }^{+} \mathrm{p}<0.05,{ }^{+++} \mathrm{p}<0.001$.

\section{Results}

\subsection{Characterization of surface morphology of Ti substrates}

As shown in Fig. 2, each surface was characterized using SEM and AFM in order to verify the surface morphology. The micrographs in Fig. 2a-h show the morphology before and after surface treatments. As compared to non-treated $\mathrm{Ti}$, the treated Ti surfaces were confirmed to have surface changes. The pristine Ti disc had a certain smooth surface. After PDA treatments, the PDA coated Ti (Ti-D) had a substantial amount of precipitated particles [23]. These results were consistent with previous studies in which PDA-coated Ti exhibited a relatively rough surface morphology as compared to uncoated polymer substrates [24]. After PEI treatment of Ti-D (Ti-DP), there was no significant change in surface morphology. After CFT grafting, the CFT immobilized Ti-DP (Ti-DPC) had partial aggregates of CFT, which confirmed the presence of the CFT layer on the Ti surface as compared to other groups (Fig. 2d). Moreover, Fig. 2e-h shows a three-dimensional surface roughness as determined by AFM analysis. The AFM imaging of each substrate was performed in the range of $10 \times 10 \mu \mathrm{m}$. The root mean squared roughness $(\mathrm{Rq})$ was calculated and is shown in Fig. 2. Nontreated Ti had a relatively smooth and plane surface morphology while treated-Ti showed a rough topology. After modification with PDA, the relative roughness of Ti-D increased about $5 \%$ as compared with the non-treated Ti [25]. The appearance of Ti-D coated with PEI changed slightly as compared to the Ti-D surface, which may have been caused by the addition of PEI chains on the surface membrane [26]. However, the CFT grafting process onto the Ti-DP surface showed a much softer surface due to binding of the CFT onto the PDA/PEI coated Ti surface. Through surface characterization, the treated-Ti was found to be successfully grafted onto the pristine Ti surface. As indicated by the zeta potential measurement, Ti-D and Ti-DP are positively charged as compared with pristine Ti (Table 2) [27]. This data clearly confirms the changes in surface morphologies and roughness upon coating.

\subsection{Characterization of wettability and elements content}

Water uptake was performed to evaluate the effectiveness of the previous surface modification protocols (Fig. 3a). Pristine Ti showed a very poor water uptake over a very long time. However, the PDA coated Ti exhibited a more hydrophilic interaction with water. The contact angle with PDA significantly decreased from $75^{\circ}$, for the non-treated $\mathrm{Ti}$, to $60^{\circ}$ for the PDA coated Ti. This data is in agreement with previous research [28]. He et al. [25] reported that contact angles for PDA coated Ti changed from $64^{\circ}$ to $37^{\circ}$. After PEI treatment, the contact angle further decreased to $58^{\circ}$, as compared to Ti-D. These results correspond well with previous studies in which PDA-coated Ti following PEI treatment showed a relatively hydrophilic surface as compared to former substrates [26]. Moreover, immobilization of CFT on the Ti-DP surface particularly decreased the water contact angle to $51^{\circ}$. The decrease in the values of water uptake was attributed to superhydrophilicity of CFT, which suggested that the CFT was successfully immobilized onto the Ti surface. To further characterize the surface elements, XPS was performed to determine the surface elemental and chemical composition of the fabricated Ti hybrid. As shown in Fig. 3b, the pristine Ti showed three major common peaks including Ti2p, O1s, and C1s. However, the Ti coated with PDA displayed an increase in N1s peak, due to the presence of amine groups along the polymer chains [24]. This result indicates that the Ti-D treatment was successful. After PEI treatment, the peaks corresponding to N1s showed the highest intensity. As a result, the N1s count increased from $5.25 \%$ to $12.77 \%$, due to PEI providing an excess of amines [15]. These results demonstrate that the PEI coated surface has a relatively higher nitrogen ratio in XPS. Subsequently, CFT was grafted on to the amine groups by carbodiimide conjugation techniques. The results showed that the elemental ratio of oxygen increased from $24.28 \%$ to $62.03 \%$ while the elemental ratio of nitrogen decreased from $12.77 \%$ to $2.96 \%$. Furthermore, peaks corresponding to S2p were found on the Ti-DPC. This indicates the existence of sulfur. These surface characterizations indicate that our fabricated $\mathrm{Ti}$ hybrids were successfully grafted with CFT. The ratios of relative atomic composition are shown in Table 1 . These results are also in good agreement with the water contact angle results.

\subsection{Quantification of amine and carboxyl groups on Ti substrates}

As described previously [29], the Acid Orange II method can give information regarding the amount of surface amine groups (Fig. 4). As PDA was attached to the pristine Ti, the grafted amount of amine increased to $13 \mu \mathrm{g}$ (Fig. 4a). After attachment of amine-rich PEI, the amount of amine groups rose to about $25 \mu \mathrm{g}$. However, the amine content of the final substrate with CFT is lower than the previous one. The amount of free amine measured was reduced by its reaction with the carboxylic acid groups on CFT forming amide bonds. Additionally, the carboxyl group on the Ti surface was measured by TBO (Fig. 4b). This confirmed that the carboxyl group increased on the final substrate as compared to pristine Ti surface due to CFT carboxylic acid groups. These results indicate that the treatment of Ti surfaces served to bind CFT to the Ti through these unique surface variations. These results display a statistically significant difference.

\subsection{Release behavior of CFT from the Ti substrates}

The release of antimicrobials from biomedical implants is an effective way to prevent bacterial infections and a rapid release is desired for a fast response time. The in vitro CFT release profiles of Ti-DPC are shown in Fig. 5. This study found drastic differences in the drug release trends under different $\mathrm{pH}$ levels. In consideration of these differences, the in vitro release of CFT was investigated at $37^{\circ} \mathrm{C}$ under the following 


\section{SEM}
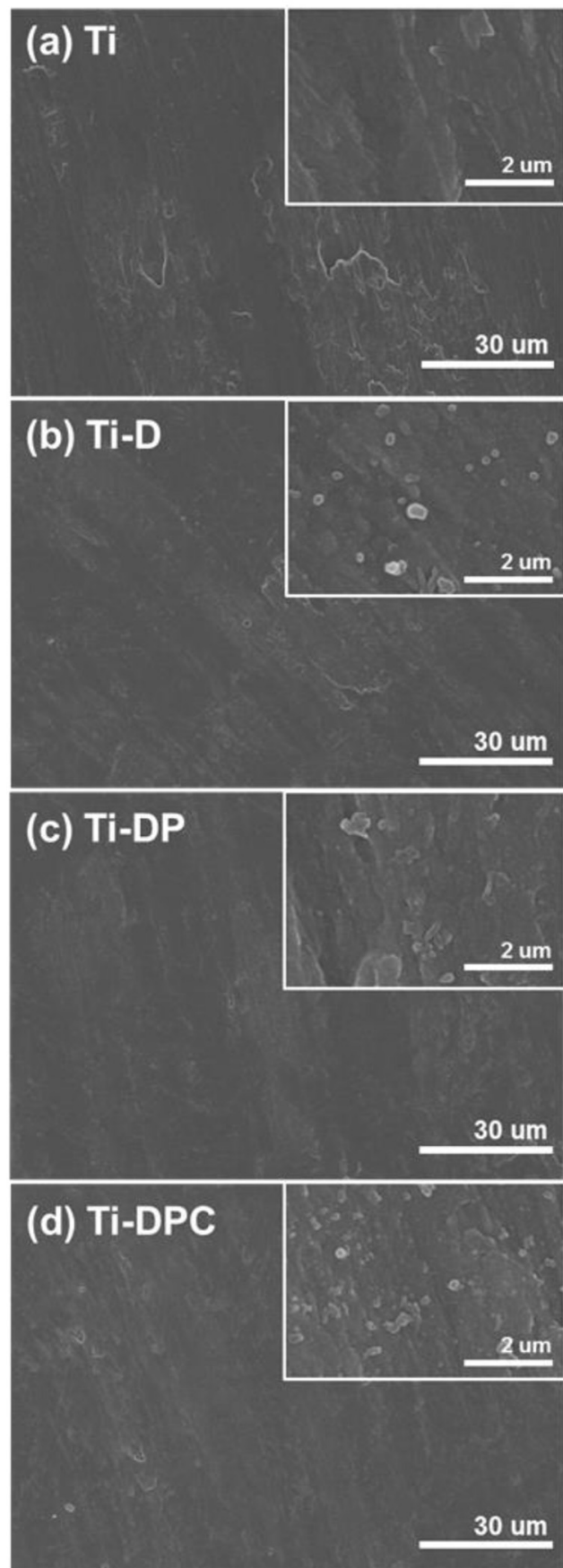

\section{AFM}

(e)



(f)

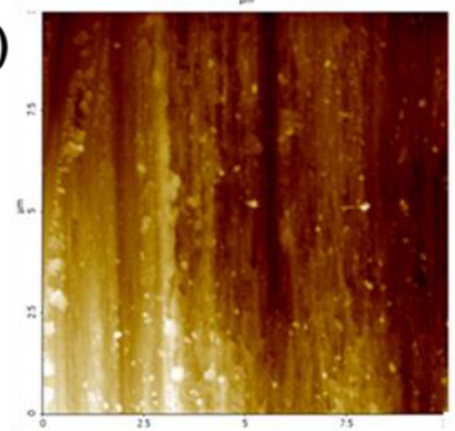

(g)

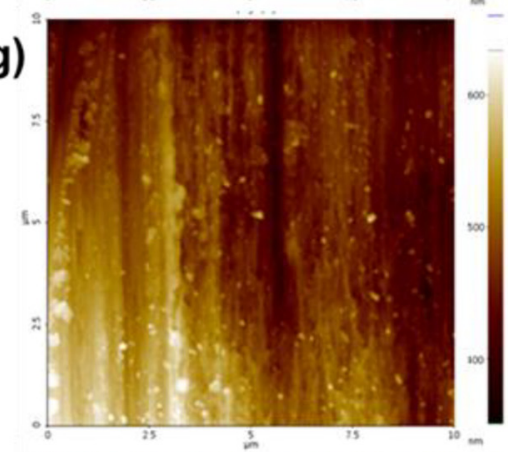

(h)

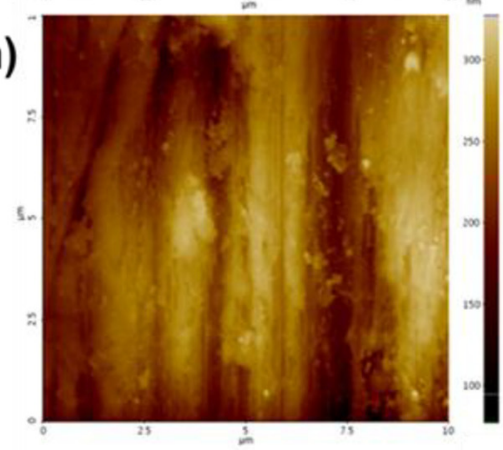

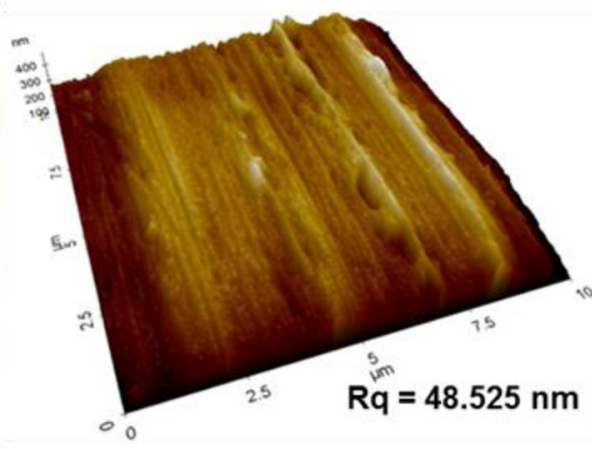
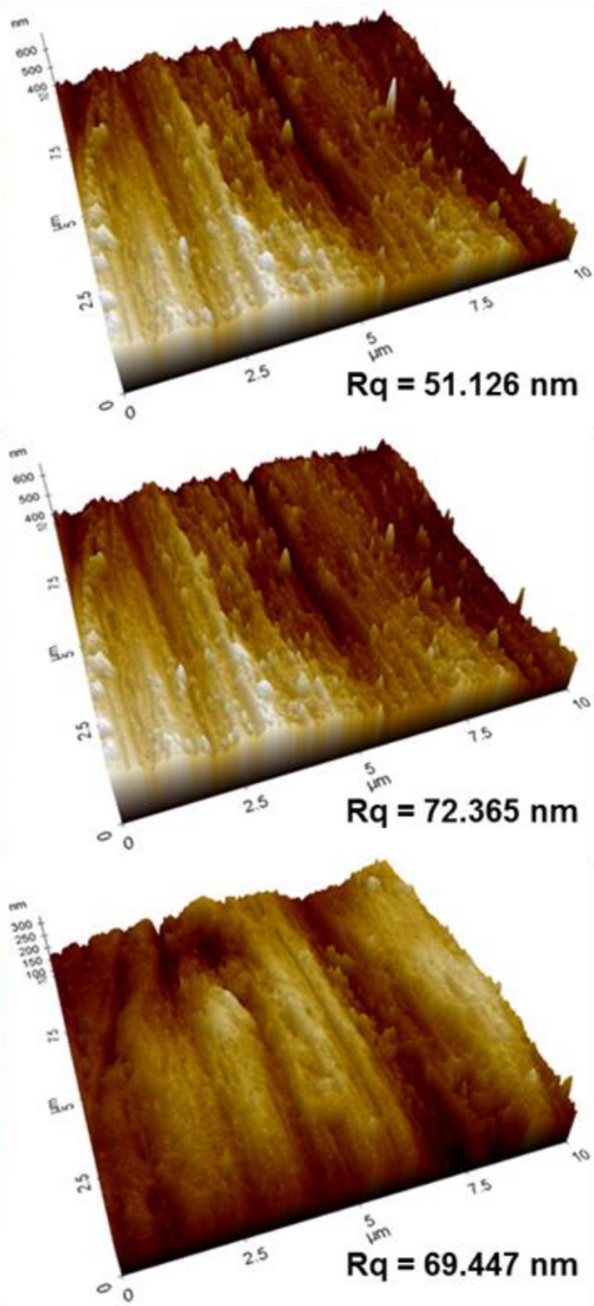

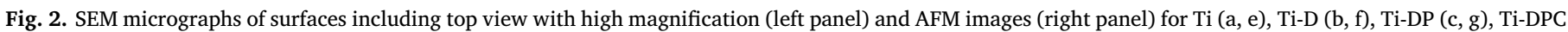
(d, h), respectively.

conditions; (1) pH 7.4, (2) pH 6.5 and (3) pH 5.5. As shown in Fig. 5, the amount of CFT released from the substrates was higher at $\mathrm{pH} 5.5$ than at $\mathrm{pH}$ 7.4. During the first early hours, there was an initial burst release of about $40 \%$ observed from the substrate. After $24 \mathrm{~h}$, approximately $80 \%$ of drug was released from the substrates at $\mathrm{pH}$ 5.5. By comparison, about $70 \%$ of drug was released at $\mathrm{pH} 6.5$ and $\mathrm{pH} 7.4$ within the same period. These results indicate that $\mathrm{pH}$ plays an important role in drug release and that the drug releases faster at acidic $\mathrm{pH}$.

\subsection{Evaluation of biocompatibility}

The cytotoxicity of the antibiotics immobilized on Ti substrates is considerably important for biomedical applications such as orthopedics and dentistry. To investigate the cytotoxicity of antibiotics immobilized on the Ti substrates, we carried out cytotoxicity assays on all test surfaces using human ADSCs at 1, 3, and 7 days of incubation. Fig. 6a shows that no cytotoxic effects were observed from these surfaces over that period of time, indicating that modification of the Ti surface do not decrease cell proliferation. The cells on pristine Ti and Ti-D showed no statistical differences in viability over 1 day. These results were 
(a)

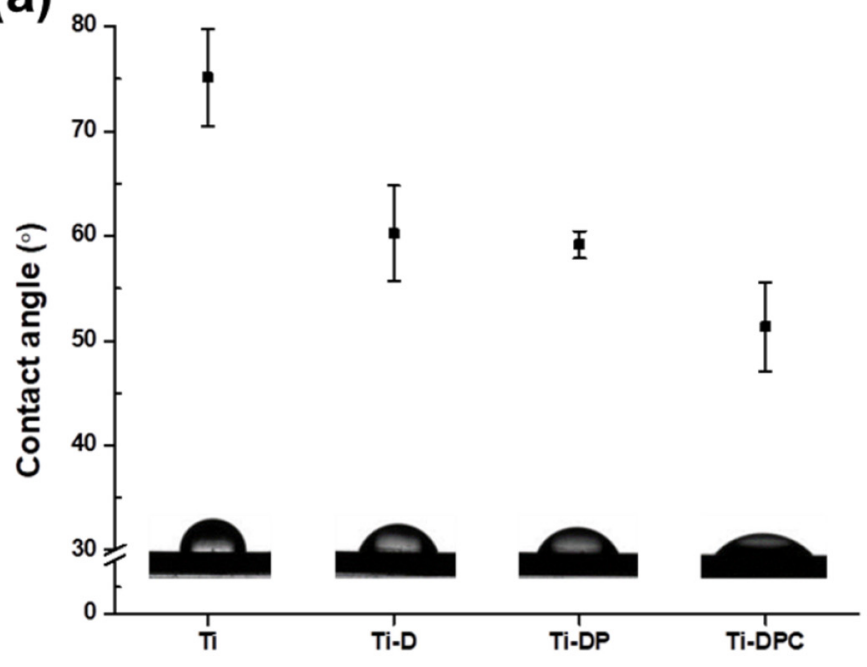

(b)

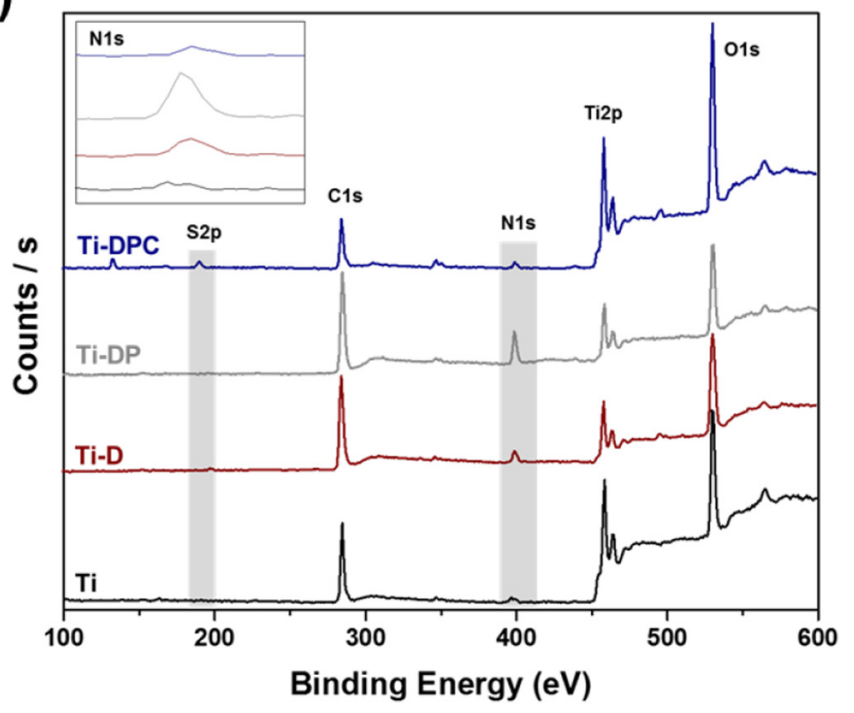

Fig. 3. Water contact angle measurement after surface treatment (a) and XPS analysis (b) for Ti, Ti-D, Ti-DP and Ti-DPC respectively.

consistent with a previous study [30]. Notably, surface modification of Ti maintains better viability and cell adhesion over time [25]. As a result, after incubating for 3 and 7 days, Ti-D, Ti-DP, and Ti-DPC showed an increase in cell proliferation rate as compared with pristine Ti. The viability of these cells displays statistically significant differences. The Ti-DPC group exhibited the highest cell proliferation rate on the seventh day. These in vitro results indicate that the adhesion of antibiotic CFT on the Ti surfaces significantly enhanced cell viability. As shown in Fig. 6b, we visualized the cell morphologies on the various functionalized Ti surfaces by SEM, Live/Dead, and CLSM images for 7 days. With each progressive surface treatment, the number of living cells was confirmed to visually increase. Interestingly, the Ti-DPC groups displayed a substantial improvement in cell viability as compared to other groups. Most of the cells were dyed green in color, which indicates that these have a possibility to survive and multiply. Also, these results were similar with those of the cell proliferation assay. These results confirmed that modification of Ti surfaces can positively influence cell adhesion improving the biocompatibility of these surfaces.

\subsection{Antibacterial property of the hybrid Ti}

The results from antibacterial activity testing are shown in Fig. 7. Each surface was tested after $24 \mathrm{~h}$ in contact with the bacterial suspension. The density and number of colonies on the Ti surfaces were evaluated. This in vitro testing of bacteria was performed against two kinds of pathogens. These were gram-negative Pseudomonas aeruginosa (P. aeruginosa), and gram-positive Methicillin-resistant Staphylococcus aureus ( $S$. aureus). These were selected as they are two major pathogenic bacteria which cause community associated infections in dentistry and orthopedics [31]. To verify the presence of bacteria after incubation, we investigated the antimicrobial action by SEM analysis.
Fig. 7a shows the bacterial growth on various substrates and the morphologies of these surfaces after bacterial challenge. The surfaces of pristine $\mathrm{Ti}$ and Ti-DP exhibited numerous bacterial colonies, while fewer bacteria were counted on the surface of Ti-DPC (Fig. 7b). Regardless of the type of bacteria, these results indicted notably strong inhibition after antibiotic surface treatments. To evaluate that the antibiotics loaded Ti discs have antimicrobial activity, the Ti-DPC was compared with the pristine Ti and Ti-DP as a control group over time. After $24 \mathrm{~h}$, more bacteria were substantially eradicated from the CFT immobilized Ti hybrids as compared to the other control groups. These tests revealed statistically significant differences (Fig. 7c). This data indicates that the antibiotic CFT was well grafted on these surfaces and effectively prohibited bacterial colony growth. Therefore, we determined that the antimicrobial Ti substrates could prevent infection by pathogenic organisms.

\section{Discussion}

Over the past several decades, implant failure is often caused by infection of the device with bacteria. This can occur during surgery or by other modes of contamination [32]. In the clinical area, the failure of Ti due to infection with bacteria has been increasing year-by-year worldwide [33]. These failures often require additional procedures to correct the implant failure which gives rise to higher healthcare costs. Accordingly, this problem comes to the forefront of both patients and surgeons. In order to solve these issues, many researchers have made an effort to develop immobilize antibiotics on Ti surfaces through chemical approaches. Unfortunately, previous methods have drawbacks due to time and cost. Furthermore, the remaining residues from the chemical reaction can cause severe toxicity which may prove to be fatal to patients.

For these reasons, we prepared a functional, antibacterial Ti using

Table 1

Atomic chemical compositions of the surfaces of the Ti, Ti-D, Ti-DP, and Ti-DPC by XPS analysis.

\begin{tabular}{|c|c|c|c|c|c|c|c|c|}
\hline \multirow[t]{2}{*}{ Atom } & \multicolumn{2}{|l|}{$\mathrm{Ti}$} & \multicolumn{2}{|l|}{ Ti-D } & \multicolumn{2}{|l|}{ Ti-DP } & \multicolumn{2}{|l|}{ Ti-DPC } \\
\hline & Peak BE & Atomic $\%$ & Peak BE & Atomic \% & Peak BE & Atomic \% & Peak BE & Atomic $\%$ \\
\hline $\mathrm{C}$ & 286.148 & 52.49 & 285.24 & 62.48 & 285.69 & 62.71 & 286.99 & 34.27 \\
\hline $\mathrm{O}$ & 531.26 & 43.03 & 531.48 & 32.02 & 530.99 & 24.28 & 531.09 & 62.03 \\
\hline $\mathrm{N}$ & 398.48 & 3.3 & 400.66 & 5.25 & 399.74 & 12.77 & 401.29 & 2.96 \\
\hline S & 163.77 & 1.16 & 162.76 & 0.26 & 162.58 & 0.24 & 169.01 & 0.74 \\
\hline
\end{tabular}


(a)

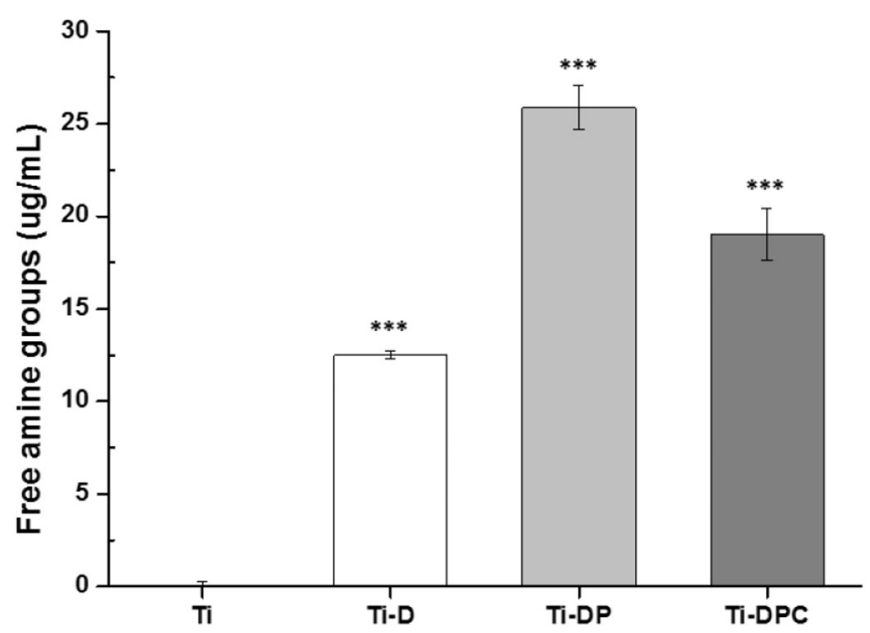

(b)

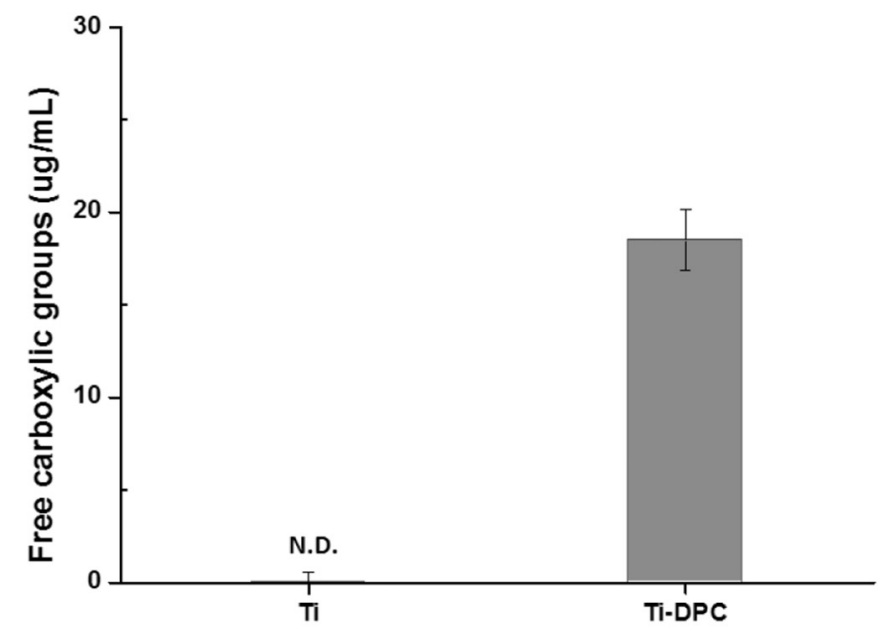

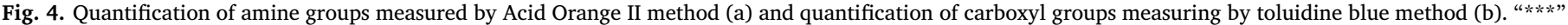
indicates significant difference of $\mathrm{p}<0.001$. N.D. means not detected.

bio-friendly materials by a convenient and facile procedure (Fig. 1). We utilized an adhesive PDA inspired by mussel adhesion chemistry in order to graft substrates. PDA is a widely used adhesive material which has catechol (DOPA) and amine (lysine) groups that can attach to all kinds of inorganic and organic surfaces [34]. Although the polymerization and deposition mechanism is clearly unknown at this time, it is likely that the adhesive-substrate interface is engaged by the catecholic units $[11,35]$. In accordance with prior research, oxidation of the catechol results in a quinone, which is structurally useful for polymerization by Schiff base or Michael additions [35]. By itself, the surface of Ti doesn't have functional groups and has limited interactions in the wet condition. For this reason, it is extremely difficult to modify the surface of $\mathrm{Ti}$ substrates. In several studies, evaporation based methods and etching techniques have been used however these are difficult, time consuming, and expensive [36]. Furthermore, these approaches may have a bad influence on the bio-functionalized $\mathrm{Ti}$ development. The use of PDA as a modification tool has advantages over these methods because of its high efficiency, low cost, and facile methodology $[37,38]$. This can be used to modify various platforms for secondary reactions, leading to tailoring of surface coatings for diverse functional uses with strong covalent and noncovalent interactions. DOPA (catechol group) attachment to the substrate surface is capable of

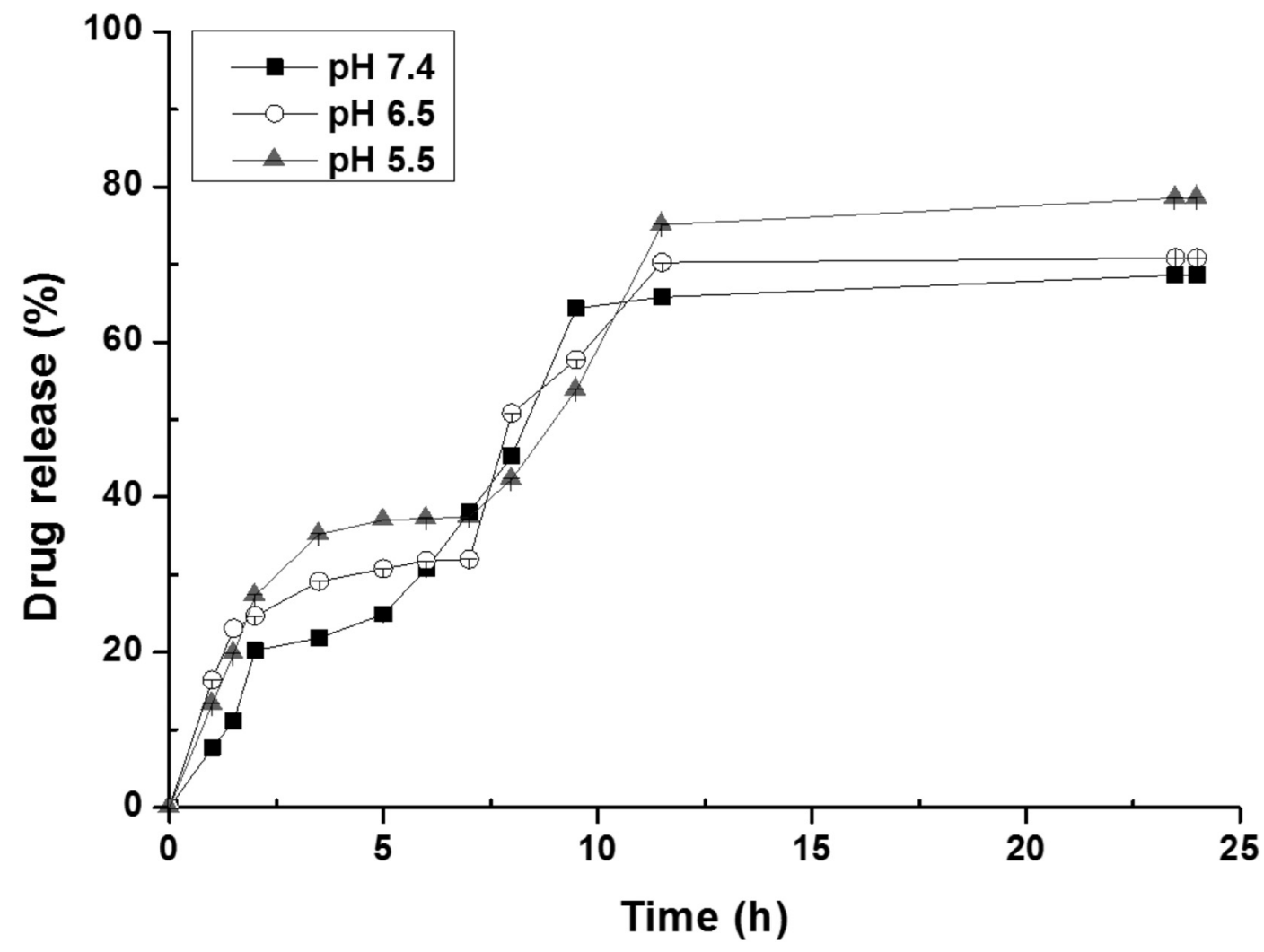

Fig. 5. Cumulative ceftazidime released from Ti-DPC at different $\mathrm{pH}$. 

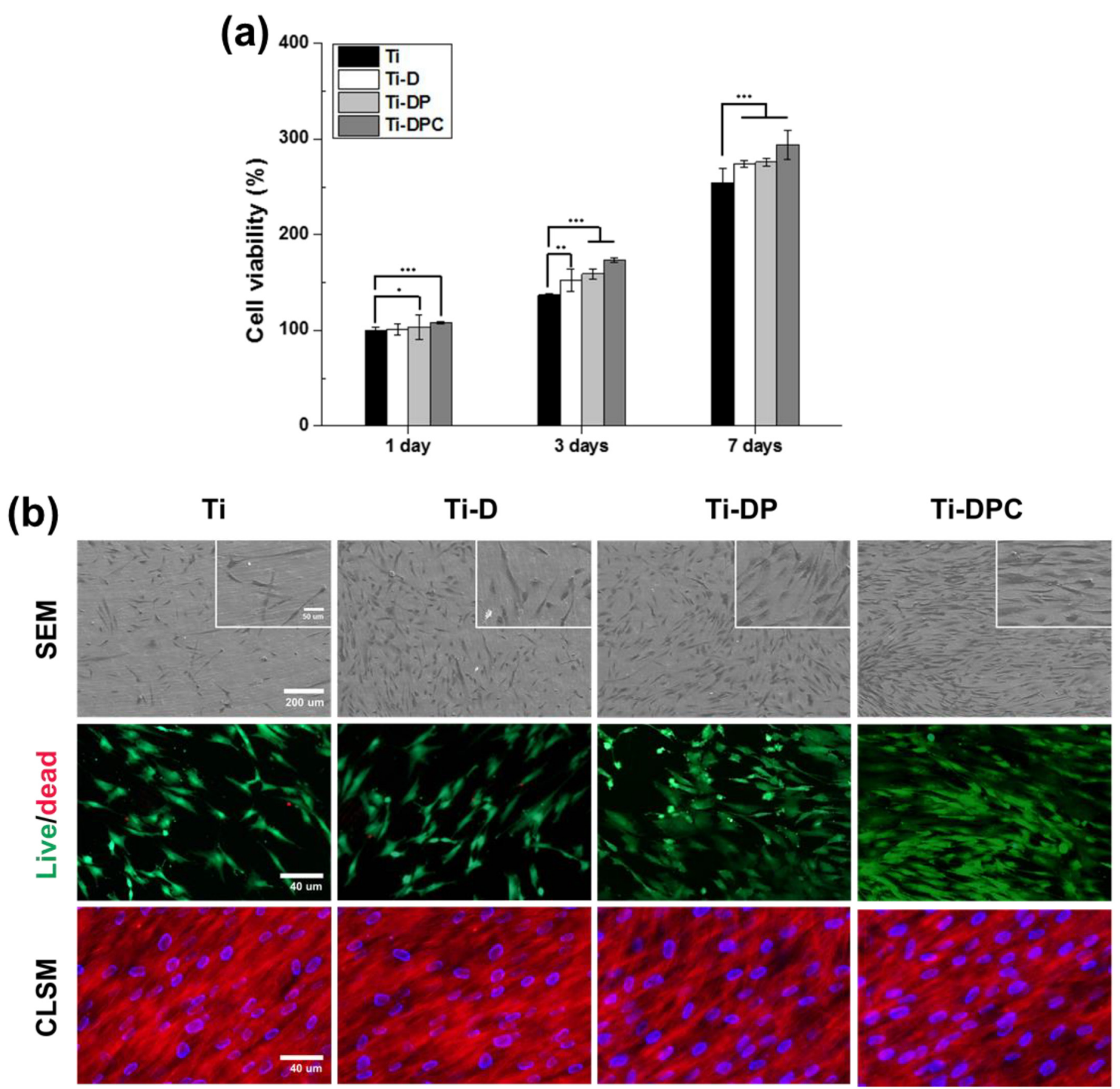

Fig. 6. Cell viability test of ADSCs for 7 days (a) and surface visualization of SEM, live \& dead, and staining on the Ti, Ti-D, Ti-DP and Ti-DPC after 7 days of culture (b). "*” indicates significant difference of $p<0.05$. “*** indicates significant difference of $p<0.01$. “****" indicates significant difference of $p<0.001$.

forming strong connections with other functional groups, like amine and thiol. Despite these advantages, PDA has insufficient amine groups in order to bind carboxylated antibiotics. Thus, we introduced more amine groups using PEI chemistry in order to enhance the binding. Amine groups from the PEI polymer conjugate to the remaining catechol groups on the surface. Specifically, the titanium surface reacts to form covalent or physical bonds with PDA. Subsequently, the catechol groups conjugate with the nucleophilic amine groups of PEI to bind the surface modification onto the Ti implant [17]. In our recent previous report, Lee et al. demonstrated that the PDA deposition of PEI coating provides a lot of opportunity for grafting of carboxyl group bearing molecules due to many amine units [19]. Through these experiments, we clearly demonstrated changes in surface composition and morphology (Fig. 2) during the surface treatments. Previously Zhang et al.
[39] developed a novel, composite nanofiltration membrane modified with PDA followed by PEI grafting. These were successively prepared as positively-charged membranes to enhance surface hydrophilicity by controlling reaction time and concentration. For this reason, we speculated that these surface modifications would increase the hydrophilicity. In the present study, our developed Ti substrates show enhanced hydrophilic surface properties through these chemical modifications (Fig. 3a). This phenomenon is due to chemical composition changes caused by increasing amine groups on the Ti surface (Fig. 3b). In this study, we found that amine content increased with the PDA/PEI surface treatments. The amount of amine groups on the surface was determined by labeling with Acid Orange II (Fig. 4a). In particular, TiDP was grafted with the largest number of amines. These amines provide for an environment allowing for cells to multiply across the 
(a)

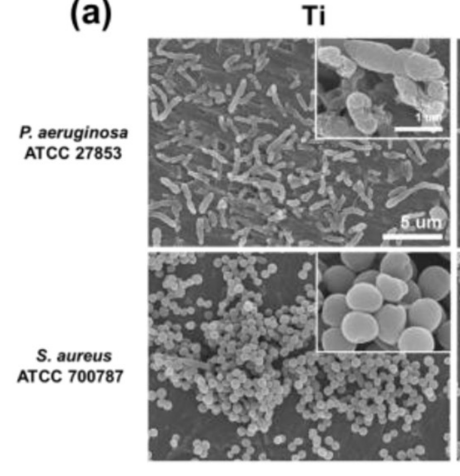

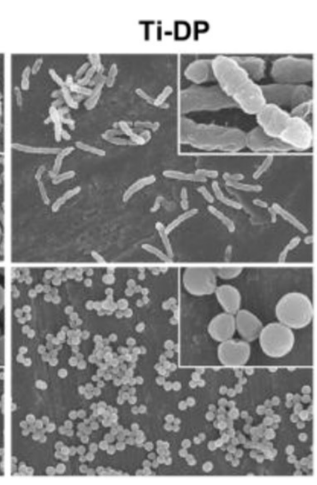

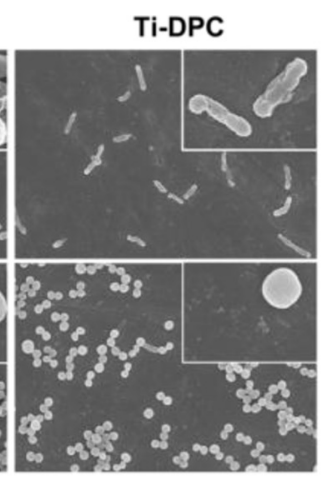

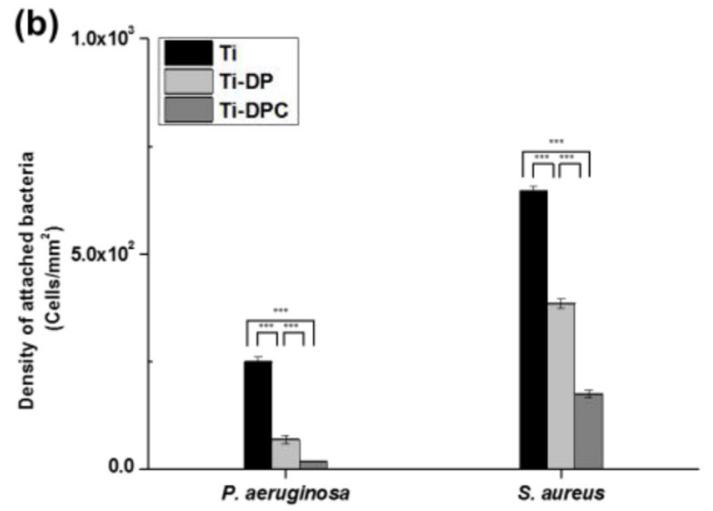

(c)

\section{P. aeruginosa ATCC 27853}

Ti

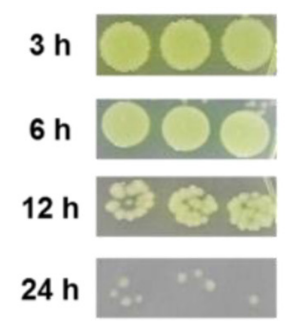

Ti-DP

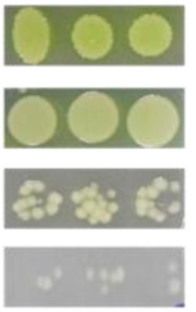

Ti-DPC

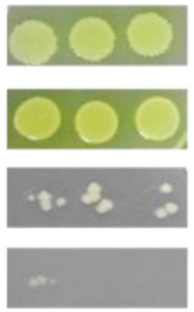

S. aureus ATCC 700787

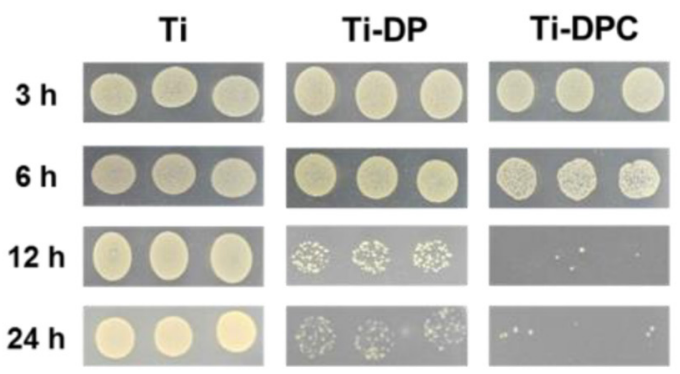

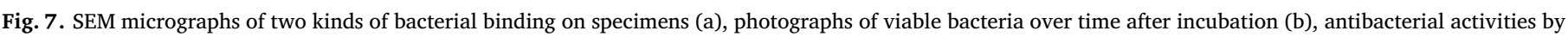

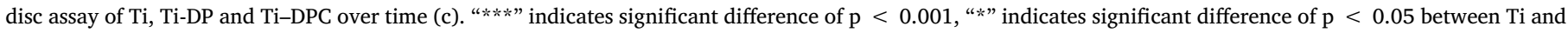
Ti-DP. " $+++"$ indicates significant difference of $\mathrm{p}<0.001$ between Ti-DP and Ti-DPC.

surfaces. Therefore, we convinced that this would improve biocompatibility and cell proliferation.

It is widely known that hydrophilic surfaces lead to cell adhesion, proliferation, differentiation, and bone formation with good success [40]. However, the majority of surfaces on Ti are generally hydrophobic, which occasionally leads to implant failure. Accordingly, this indicates that the surface of the Ti can significantly influence cell behavior. Especially, surface wettability can have a major effect on the surface-biology interface [40]. The initial effects of both cellular and tissue responses to biomaterials are mainly due to blood contact as blood proteins and molecules are the first to interact with the surface. Subsequently, the hydrophilic surface of Ti increases the formation of a fibrin blood clot which interacts with cells. These osteoblast cells affect differentiation, extracellular matrix production, and tissue regeneration. Thus, hydrophilic surface modifications can assist in the early healing period and enhance integration [41]. Similarly, our cell viability results indicated that the PDA/PEI coating also showed excellent cell proliferation as compared to bare Ti (Fig. 6).

As is well known, Ti alone doesn't have antibacterial effect and tends to form a surface protein layer under physiological conditions. This protein layer is suitable for bacterial colonization [42]. To interfere with the formation of a biofilm on the Ti surface, there are two methods which can be applied at the initial and most vulnerable stages [40]. First, anti-adhesive coatings on surfaces are applied to impede the formation of the biofilm. These substrates consist of surface modifications, such as PDA, and cationic polymers, like PEI. In a previous report, PDA coating has been found to be effective for providing antimicrobial activity against biofilms [43]. A previous report by Iqbal et al. [44] suggested that encapsulation of bacteria in the PDA film also inhibited bacterial proliferation and survival. In another study, positively charged polymers, such as PEI, can enhance permeability of negatively charged bacterial outer membranes $[45,46]$. It is considered that the PEI chemistry on the titanium surfaces alone can also inhibit bacterial activity [47]. As indicated in previous research, the positive charge of PEI due to the protonation of the primary, secondary, and tertiary amine groups can facilitate binding to negatively charged bacteria through electrostatic interactions [47]. After attaching to the negatively-charged surface of bacteria, PEI increases the permeability of the membrane resulting in elimination of biofilm. The utilization of cationic polymers can also help alleviate, eliminate, or eradicate microbial infections as a form of antibiotic treatment [48]. Additionally, previous reports established that PDA/PEI modified membranes clearly have an influence on anti-fouling and antibacterial properties in comparison with unmodified membranes [26]. Each additional surface treatment was found to improve the antimicrobial effect. As indicated by the zeta potential measurement, PDA/PEI coatings are positively charged, which can induce inhibition of biofilm formation (Table 2) [27]. Therefore, we expected to effectively eradicate bacteria by introducing PDA/PEI coatings. Second, antibiotic agents immobilized on the surfaces can be an adequate measure to prevent infection. The choice of antibiotics is also important for the medical field. Amongst the many kinds of antibiotics, beta-lactam antimicrobial agents are one of the oldest and most popular antimicrobials. The ring structure of betalactam includes several classes of antimicrobial agents such as penicillin's and cephalosporins. As compared to penicillin's, the cephalosporin antibiotic agents have lower toxicity and more powerful

Table 2

Surface charge of the surfaces of the Ti, Ti-D, Ti-DP, and Ti-DPC by zeta potential analysis.

\begin{tabular}{lc}
\hline Surface treatment & Zeta Potential $(\mathrm{mV})$ \\
\hline $\mathrm{Ti}$ & $-4.41 \pm 0.79$ \\
$\mathrm{Ti}-\mathrm{D}$ & $-0.76 \pm 0.05$ \\
$\mathrm{Ti}-\mathrm{DP}$ & $0.35 \pm 0.70$ \\
Ti-DPC & $-2.09 \pm 0.70$ \\
\hline
\end{tabular}


antibiotic activity [49]. In this study, we employed the third generation of cephalosporin to cope with more severe gram-negative bacteria. The third-generation compounds have a greater spectrum against gram positive and negative bacteria and are effective against in vitro gramnegative organisms. This is especially true for those with beta-lactamase enzymes as compared to first and second generation drugs [50]. The basic mechanism proposed for the antibacterial action of thirdgeneration CFT is not yet fully understood. However, many groups have described that CFT works by interfering with synthesis of the peptidoglycan layer forming the bacterial cell wall [18]. Due to CFT's broad spectrum antibiotic activity against both gram negative and positive organisms, it presents a promising way to inhibit the growth of bacteria. In the present study, we used CFT because it is generally applied for medical uses and has potent antimicrobial activity against Pseudomonas (Fig. 7).

Many bacteria are known to excrete acid and lower the $\mathrm{pH}$ level of the surrounding environment [51]. The bacteria used in our experiments also lower the $\mathrm{pH}$. Since the $\mathrm{pH}$ environment plays an important role, the activity of drug release under different $\mathrm{pH}$ conditions was explored. The release of drugs from the Ti surface occurs by two mechanisms. First, drug carrier polymers are affected by $\mathrm{pH}$ which aids in release of the drug. Previously, PEI polymers were found to protonate when the $\mathrm{pH}$ was lower than neutral [52]. These PEIs act to release the drug at acidic environments faster due to this protonation. Dong et al. [53] suggested that PEI-doxorubicin conjugates can be utilized as $\mathrm{pH}$ sensitive drug carriers for targeting the acidic tumor tissues (pH 6.5-6.8). Second, adequate $\mathrm{pH}$ conditions can be an appropriate means to cleave amide bonds. CFT bonds were cleaved from the substrates in response to the acidic environment. This led to a higher release of antibiotics from the substrate as compared to the release found in physiological $\mathrm{pH}$ conditions. This occurs as the hydrolysis of amide bonds occurs more readily at lower $\mathrm{pH}$ [54]. Specifically, CFT was released from the Ti-DPC more rapidly under acidic $\mathrm{pH}$ conditions (5.5). This is is due to cleavage of the labile amide bond. It has been previously demonstrated that the amide bond formed between PEI and CFT is prone to $\mathrm{pH}$-dependent hydrolysis [55]. This result (Fig. 5) confirms that drugs are released faster in low $\mathrm{pH}$ environments. Since bacteria act to lower the $\mathrm{pH}$ conditions of the surrounding environment, this is representative of the bacterial habitat environment. In this environment, the release of antibiotics from the surface of Ti was particularly important for the antimicrobial action. The most appropriate measurement time and methods for determining antibacterial effect has been discussed for several years. In previous reports, Alasdair et al. proposed to measure IE (intensity of effect) as the optimal measurement method. In the case of exposure to antibiotics, this measurement is determined by comparing the control bacterial growth curve with the test curve. Under this method, the test group is evaluated over the course of the initial inhibition period and during regeneration to reach the same density as the control after the control phase. Generally this occurs after 24 or $48 \mathrm{~h}$ and the evaluation criteria is the time to kill $99.9 \%$ or $99 \%$ of the initial inoculum and the resultant changes in survival count $(\log \mathrm{cfu} / \mathrm{ml})$ [56]. In the absence of antibacterial effect, implantation may create an opening for bacterial invasion leading to peri-implantitis and nosocomial infections. In this situation, the inhibition of the bacteria within the earlier time points that occur immediately after surgical implantation is key to prevention fatal infections [57]. In this study, we focused on and carried out only preliminary assessments to confirm if the material could be applied to clinical applications. However, almost all bacteria were substantially eradicated from the CFT immobilized Ti hybrids after about $12 \mathrm{~h}$. Over this time, approximately $80 \%$ of the CFT was released from the substrates. In this study, the cleavage of amide bonds in acidic environments would promote the release of antimicrobial agents, eventually preventing bacteria adhesion and improving antibacterial effects. This remarkable result indicates the great potential of $\mathrm{pH}$-responsive $\mathrm{Ti}$ substrates as drug carriers. These provide for a rapid and controlled release of antibiotics.

Overall, the development of Ti substrates grafted with CFT using the PDA/PEI coating represents a desirable approach for biomedical applications. We expect that application of both anti-adhesive coating and antibiotics can provide for simultaneous and synergistic efficacy. In this study, we determined these substrates to have both bioactive and antimicrobial results through in vitro assessment. In this case, our developed substrates provide for a potential tool for use in clinical treatment.

\section{Conclusion}

In summary, we designed and prepared a hybrid Ti surface including a PDA/PEI coating followed by grafting of an antibiotic agent, CFT in order to enhance its biocompatibility and antibacterial activity against pathogens through a facile synthesis method. We confirmed the novel modification of Ti surface via physicochemical assessments. The In vitro biological analysis showed that the CFT immobilized Ti had good antibacterial activity. These findings indicate that this biocompatible Ti surface can be used as an effective tool for the generation of an antimicrobial implant to eradicate bacterial infections. Furthermore, we confirmed an osteogenic possibility through loading of cephalosporin antibiotic such as CFT (Fig. S1) [58]. Based on this finding, we will do an in vivo animal study to treat osteomyelitis in the near future. This preliminary study will pave the way for future investigations. Therefore, our developed Ti surface may play a role in delivery of antibiotics which can reduce the incidence of infection for $\mathrm{Ti}$ implants.

Supplementary data to this article can be found online at https:// doi.org/10.1016/j.apsusc.2019.143675.

\section{Declaration of competing interest}

The authors declare no competing financial interest.

\section{Acknowledgement}

This research was supported by the Bio \& Medical Technology Development Program of the National Research Foundation (NRF) funded by the Korean government (MSIT) (No. 2017M3A9E4048170).

\section{References}

[1] S. Bauer, P. Schmuki, K. von der Mark, J. Park, Engineering biocompatible implant surfaces: part I: materials and surfaces, Prog. Mater. Sci. 58 (2013) 261-326.

[2] K.M. Buettner, A.M. Valentine, Bioinorganic chemistry of titanium, Chem. Rev. 112 (2011) 1863-1881.

[3] S. Ferraris, S. Spriano, Antibacterial titanium surfaces for medical implants, Mater Sci Eng C Mater Biol Appl, Materials for biological applications 61 (2016) 965-978.

[4] W.J. Metsemakers, M. Morgenstern, M.A. McNally, T.F. Moriarty, I. McFadyen, M. Scarborough, N.A. Athanasou, P.E. Ochsner, R. Kuehl, M. Raschke, O. Borens, Z. Xie, S. Velkes, S. Hungerer, S.L. Kates, C. Zalavras, P.V. Giannoudis, R.G. Richards, M.H.J. Verhofstad, Fracture-related infection: a consensus on definition from an international expert group, Injury 49 (2018) 505-510.

[5] P.A. Norowski Jr., J.D. Bumgardner, Biomaterial and antibiotic strategies for peri implantitis: a review, J. Biomed. Mater. Res. Part B Appl. Biomater. 88 (2009) $530-543$.

[6] D. Lindner, K. Schlothofer-Schumann, B.C. Kern, O. Marx, A. Muns, J. Meixensberger, Cranioplasty using custom-made hydroxyapatite versus titanium: a randomized clinical trial, J. Neurosurg. 126 (2017) 175-183.

[7] D. Campoccia, L. Montanaro, C.R. Arciola, A review of the clinical implications of anti-infective biomaterials and infection-resistant surfaces, Biomaterials 34 (2013) 8018-8029.

[8] M. Zilberman, J.J. Elsner, Antibiotic-eluting medical devices for various applications, J. Control. Release 130 (2008) 202-215.

[9] F.J. Weber, J.A. de Bont, Adaptation mechanisms of microorganisms to the toxic effects of organic solvents on membranes, Biochim. Biophys. Acta Biomembr. 1286 (1996) 225-245.

[10] H. Lee, S.M. Dellatore, W.M. Miller, P.B. Messersmith, Mussel-inspired surface chemistry for multifunctional coatings, Science 318 (2007) 426-430.

[11] Q. Ye, F. Zhou, W. Liu, Bioinspired catecholic chemistry for surface modification, Chem. Soc. Rev. 40 (2011) 4244-4258.

[12] S. Yuan, D. Wan, B. Liang, S.O. Pehkonen, Y.P. Ting, K.G. Neoh, E.T. Kang, Lysozyme-coupled poly(poly(ethylene glycol) methacrylate)-stainless steel hybrids 
and their antifouling and antibacterial surfaces, Langmuir 27 (2011) 2761-2774.

[13] I.H. Bae, I.K. Park, D.S. Park, H. Lee, M.H. Jeong, Thromboresistant and endothelialization effects of dopamine-mediated heparin coating on a stent material surface, J Mater Sci Mater Med 23 (2012) 1259-1269.

[14] S.N. Kang, C. Park, S.M. Kim, K.W. Park, B.J. Park, D.K. Han, Y.K. Joung, Effect of stromal cell derived factor-1 $\alpha$ release from heparin-coated Co-Cr stent substrate on the recruitment of endothelial progenitor cells, Macromol. Res. 23 (2015) 1159-1167.

[15] R. Luo, X. Wang, J. Deng, H. Zhang, M.F. Maitz, L. Yang, J. Wang, N. Huang, Y. Wang, Dopamine-assisted deposition of poly (ethylene imine) for efficient heparinization, Colloids Surf B Biointerfaces 144 (2016) 90-98.

[16] H. Wang, M. Ling, Y. Bai, S. Chen, Y. Yuan, G. Liu, C. Wu, F. Wu, Cationic polymer binder inhibit shuttle effects through electrostatic confinement in lithium sulfur batteries, J Mater Chem A Mater 6 (2018) 6959-6966.

[17] C. Zhao, F. Zuo, Z. Liao, Z. Qin, S. Du, Z. Zhao, Mussel-inspired one-pot synthesis of a fluorescent and water-soluble polydopamine-polyethyleneimine copolymer, Macromol. Rapid Commun. 36 (2015) 909-915.

[18] D. Zhang, Y. Wang, S. Ma, S. Wu, H. Hao, Determination of solubility and induction time of ceftazidime, J. Chem. Eng. Data 58 (2012) 176-182.

[19] S.J. Lee, H.H. Jo, K.S. Lim, D. Lim, S. Lee, J.H. Lee, W.D. Kim, M.H. Jeong, J.Y. Lim, I.K. Kwon, Y. Jung, J.K. Park, S.A. Park, Heparin coating on 3D printed poly (1-lactic acid) biodegradable cardiovascular stent via mild surface modification approach for cornary artery implantation, Chem. Eng. J. 378 (2019) 1-15.

[20] D.N. Heo, W.K. Ko, H.R. Lee, S.J. Lee, D. Lee, S.H. Um, J.H. Lee, Y.H. Woo, L.G. Zhang, D.W. Lee, I.K. Kwon, Titanium dental implants surface-immobilized with gold nanoparticles as osteoinductive agents for rapid osseointegration, J. Colloid Interface Sci. 469 (2016) 129-137.

[21] J.H. Moon, E.Y. Jang, K.S. Shim, J.Y. Lee, In vitro effects of $\mathrm{N}$-acetyl cysteine alone and in combination with antibiotics on Prevotella intermedia, J. Microbiol. 53 (2015) 321-329.

[22] E.Y. Jang, M. Kim, M.H. Noh, J.H. Moon, J.Y. Lee, In vitro effects of polyphosphate against Prevotella intermedia in planktonic phase and biofilm, Antimicrob. Agents Chemother. 60 (2016) 818-826.

[23] J. Jiang, L. Zhu, L. Zhu, B. Zhu, Y. Xu, Surface characteristics of a self-polymerized dopamine coating deposited on hydrophobic polymer films, Langmuir 27 (2011) 14180-14187.

[24] H.J. Cho, S.K. Perikamana, J.H. Lee, J. Lee, K.M. Lee, C.S. Shin, H. Shin, Effective immobilization of BMP-2 mediated by polydopamine coating on biodegradable nanofibers for enhanced in vivo bone formation, ACS Appl. Mater. Interfaces 6 (2014) 11225-11235.

[25] S. He, P. Zhou, L. Wang, X. Xiong, Y. Zhang, Y. Deng, S. Wei, Antibiotic-decorated titanium with enhanced antibacterial activity through adhesive polydopamine for dental/bone implant, J. R. Soc. Interface 11 (2014) 20140169.

[26] H. Li, L. Peng, Y. Luo, P. Yu, Enhancement in membrane performances of a commercial polyamide reverse osmosis membrane via surface coating of polydopamine followed by the grafting of polyethylenimine, RSC Adv. 5 (2015) 98566-98575.

[27] X. Li, J. Deng, S. Yuan, J. Wang, R. Luo, S. Chen, J. Wang, N. Huang, Fabrication of endothelial progenitor cell capture surface via DNA aptamer modifying dopamine/ polyethyleneimine copolymer film, Appl. Surf. Sci. 386 (2016) 138-150.

[28] Q. Wei, B. Li, N. Yi, B. Su, Z. Yin, F. Zhang, J. Li, C. Zhao, Improving the blood compatibility of material surfaces via biomolecule-immobilized mussel-inspired coatings, J. Biomed. Mater. Res. A 96 (2011) 38-45.

[29] S. Noel, B. Liberelle, L. Robitaille, G. De Crescenzo, Quantification of primary amine groups available for subsequent biofunctionalization of polymer surfaces, Bioconjug. Chem. 22 (2011) 1690-1699.

[30] K. Yang, J.S. Lee, J. Kim, Y.B. Lee, H. Shin, S.H. Um, J.B. Kim, K.I. Park, H. Lee, S.W. Cho, Polydopamine-mediated surface modification of scaffold materials for human neural stem cell engineering, Biomaterials 33 (2012) 6952-6964.

[31] R. Hernandez, The use of systemic antibiotics in the treatment of chronic wounds, Dermatol. Ther. 19 (2006) 326-337.

[32] M. Salwiczek, Y. Qu, J. Gardiner, R.A. Strugnell, T. Lithgow, K.M. McLean, H. Thissen, Emerging rules for effective antimicrobial coatings, Trends Biotechnol. 32 (2014) 82-90.

[33] J. Lindhe, J. Meyle, D.o.E.W.o.P. Group, Peri-implant diseases: consensus report of the sixth European workshop on periodontology, J. Clin. Periodontol. 35 (2008) 282-285.

[34] M.E. Lynge, R. van der Westen, A. Postma, B. Städler, Polydopamine-a natureinspired polymer coating for biomedical science, Nanoscale 3 (2011) 4916-4928.

[35] E. Faurea, C.F. Daudré, C. Jérôme, J. Lyskawa, D. Fournier, P. Woisel,
C. Detrembleur, Catechols as versatile platforms in polymer chemistry, Prog. Polym. Sci. 38 (2013) 236-270.

[36] A. Sharonova, M. Surmeneva, K. Loza, O. Prymak, M. Epple, R. Surmenv, Surface functionalization of titanium with silver nanoparticles, J. Phys. Conf. Ser. 1145 (2019) 1-7.

[37] Z.Y. Xi, Y.Y. Xu, L.P. Zhu, Y. Wang, B.K. Zhu, A facile method of surface modification for hydrophobic polymer membranes based on the adhesive behavior of poly(DOPA) and poly(dopamine), J Memb Sci 327 (2009) 244-253.

[38] J. Wu, C. Cai, Z. Zhou, H. Qian, F. Zha, J. Guo, B. Feng, T. He, N. Zhao, J. Xu, Lowcost mussel inspired poly(catechol/polyamine) coating with superior anti-corrosion capability on copper, J. Colloid Interface Sci. 462 (2016) 214-221.

[39] R. Zhang, Y. Su, X. Zhao, Y. Li, J. Zhao, Z. Jiang, A novel positively charged composite nanofiltration membrane prepared by bio-inspired adhesion of polydopamine and surface grafting of poly(ethylene imine), J Memb Sci 470 (2014) 9-17.

[40] R.A. Gittens, L. Scheideler, F. Rupp, S.L. Hyzy, J. Geis-Gerstorfer, Z. Schwartz, B.D. Boyan, A review on the wettability of dental implant surfaces II: biological and clinical aspects, Acta Biomater. 10 (2014) 2907-2918.

[41] W. Degasperi, P. Andersson, D. Verrocchi, L. Sennerby, One-year clinical and radiographic results with a novel hydrophilic titanium dental implant, Clin. Implant. Dent. Relat. Res. 16 (2014) 511-519.

[42] L. Zhao, P.K. Chu, Y. Zhang, Z. Wu, Antibacterial coatings on titanium implants, J. Biomed. Mater. Res. Part B Appl. Biomater. 91 (2009) 470-480.

[43] Y.H. Ding, M. Floren, W. Tan, Mussel-inspired polydopamine for bio-surface functionalization, Biosurf Biotribol 2 (2016) 121-136.

[44] Z. Iqbal, E.P.C. Lai, T.J. Avis, Antimicrobial effect of polydopamine coating on Escherichia coli, J. Mater. Chem. 22 (2012) 21608.

[45] S.R. Clark, K.Y. Lee, H. Lee, J. Khetan, H.C. Kim, Y.H. Choi, K. Shin, Y.Y. Won, Determining the effects of PEI adsorption on the permeability of 1,2-dipalmitoylphosphatidylcholine/bis(monoacylglycero)phosphate membranes under osmotic stress, Acta Biomater. 65 (2018) 317-326.

[46] H. Khalil, T. Chen, R. Riffon, R. Wang, Z. Wang, Synergy between polyethylenimine and different families of antibiotics against a resistant clinical isolate of Pseudomonas aeruginosa, Antimicrob. Agents Chemother. 52 (2008) 1635-1641.

[47] Z. Liu, Y. Wang, Y. Zu, Y. Fu, N. Li, N. Guo, R. Liu, Y. Zhang, Synthesis of polyethylenimine (PEI) functionalized silver nanoparticles by a hydrothermal method and their antibacterial activity study, Mater Sci Eng C 42 (2014) 31-37.

[48] S.K. Samal, M. Dash, S. Van Vlierberghe, D.L. Kaplan, E. Chiellini, C. van Blitterswijk, L. Moroni, P. Dubruel, Cationic polymers and their therapeutic potential, Chem. Soc. Rev. 41 (2012) 7147-7194.

[49] E. Abraham, G. Newton, Penicillins and cephalosporins, Biosynthesis, Springer, Berlin, 1967, pp. 1-16.

[50] T.J. Dougherty, M.J. Pucci, Antibiotic Discovery and Development, Springe Science \& Business Media, Boston, 2011.

[51] L. Zhang, F. Su, X. Kong, F. Lee, K. Day, W. Gao, M.E. Vecera, J.M. Sohr, S. Buizer Y. Tian, D.R. Meldrum, Ratiometric fluorescent $\mathrm{pH}$-sensitive polymers for highthroughput monitoring of extracellular pH, RSC Adv. 6 (2016) 46134-46142.

[52] X. Guan, Y. Li, Z. Jiao, J. Chen, Z. Guo, H. Tian, X. Chen, A pH-sensitive chargeconversion system for doxorubicin delivery, Acta Biomater. 9 (2013) 7672-7678.

[53] D.W. Dong, S.W. Tong, X.R. Qi, Comparative studies of polyethylenimine-doxorubicin conjugates with $\mathrm{pH}$-sensitive and $\mathrm{pH}$-insensitive linkers, J. Biomed. Mater. Res. A 101 (2013) 1336-1344.

[54] R.M. Smith, D.E. Hansen, The pH-rate profile for the hydrolysis of a peptide bond, J. Am. Chem. Soc. 120 (1998) 8910-8913.

[55] L. Wu, Y. Zou, C. Deng, R. Cheng, F. Meng, Z. Zhong, Intracellular release of doxorubicin from core-crosslinked polypeptide micelles triggered by both $\mathrm{pH}$ and reduction conditions, Biomaterials 34 (2013) 5262-5272.

[56] A. MacGowan, C. Rogers, H.A. Holt, M. wootton, K. Bowker, Assessment of different antibacterial effect measures used in vitro models of infection and subsequent use in pharmacodynamic correlations for moxifloxacin, J. Antimicrob. Chemother. 46 (2000) 73-78.

[57] S.J. Lee, D.N. Heo, H.R. Lee, D. Lee, S.J. Yu, S.A. Park, W.K. Ko, S.W. Park, S.G. Im, J.H. Moon, I.K. Kwon, Biofunctionalized titanium with anti-fouling resistance by grafting thermo-responsive polymer brushes for the preventionof peri-implantitis, $\mathrm{J}$ Mater. Chem. B 3 (2015) 5161-5165.

[58] C.R. Rathbone, J.D. Cross, K.V. Brown, C.K. Murray, J.C. Wenke, Effect of various concentrations of antibiotics on osteogenic cell viability and activity, J. Orthop. Res. 29 (2011) 1070-1074. 